\title{
XIX.
}

\section{Über einen Fall von Poliomyelitis anterior chronica mit Sektionsbefund.}

\author{
Von \\ Dr. T. Aoyama, \\ Professor an der Universität in Tokio.
}

Seit den Mitteilungen von Oppenheim und Nonne über Poliomyelitis anterior chronica ist mehr als ein Dezennium verflossen and immer noch sind die Berichte über diese Krankheit sehr spärlich. Ich glaube deshalb, dass die nachfolgende kasuistische Mitteilung nicht überflüssig sein wird, wenn damit auch nicht viel Neues zu dem jetzt Bekannten hinzugefügt wird.

Ich schicke die Krankengeschichte und den Sektionsbericht der Besprechung des Falles voraus.

Anamnese: Osawa, Arzt, 36 Jahre alt. Vater im 59. Lebensjahre an Dysenterie gestorben. Mutter gesund; ebenso alle 5 lebenden Geschwister. Ein alterer: Bruder starb mit 9 Jahren an Typhus, der Grossvater väterlicherseits an Altersschwäche. Grossmutter väterlicherseits war epileptisch. Die Ursache ihres Todes ist nicht bekannt. Grossvater mütterlicherseits starb an einer Krankheit, bei der Schluckbeschwerden auftraten. Die Todesursache der Grossmutter mütterlicherseits, die in hohem Alter starb, ist nicht bekannt.

Patient hat als Kind die Masern gehabt. Im 20. Lebensjahre bekam er Gonorrhoe, woran er ein Jahr lang litt. Syphilis negiert. Im Jahre 1896 soll er Distoma spathulatum gehabt haben.

Der Beginn der Krankheit fällt in den Januar 1901, wo zuerst beim Gehen ein spannender und ziehender Schmerz an der linken Wade auftrat, welche auch Druckempfindlichkeit aufwies. Da man den Schmerz für rheumatisch hielt, wurde Natrium salicylicum verordnet, was aber ohne Erfolg blieb. Gleichzeitig zeigte sich nun kleinschlägiges, unwillkürliches Zittern an den Fingern beider Hände, das bei kleinen Hantierungen besonders hervortrat. Am 14. März kam er auf der Strasse dreimal nach einander zu Falle, ohne mit den Füssen auf Hindernisse gestossen zu sein. Während er nach dem ersten Sturz nur Kraftlosigkeit am linken Knie verspürte, konnte er das letzte Mal nicht mehr aufstehen und musste mittelst eines Wagens nach Hause geschafft werden. Am nächsten Morgen konnte er gehen, doch war das Ermüdungsgefühl des linken Knies nicht verschwunden; 
auch bemerkte er damals zuerst, dass die Dorsalflexion der linken grossen Zehe Schwierigkeiten machte. Im April beobachtete er das erste Mal Atrophie der Peronealmuskeln. Im Mai wurde die Flexion des linken Kniegelenks schwierig und der Gang etwas hinfüllig. Etwa um diese Zeit fiel es dem Patienten auf, dass die rechte Schulter nicht mehr gehörig gehoben werden konnte. Im Juni wurde die Dorsalflexion der rechten Zehe unmöglich. Im Oktober nun zeigte sich an der linken Schulter eine Parese, die sich von da anf den Oberarm ausbreitete. Dieser Lähmung folgte eine Abmagerung, doch soll es angeblich dem Patienten eine Zeit lang möglich gewesen sein, sich an den Beinen allmählich emporzurichten, wie man es bei der myopathischen Muskelatrophie zu finden pflegt. Am Jahresende kam noch Atrophie der Brustmuskeln hinzu.

Status: Patient fand am 2.11. 1901 in der Klinik des Prof. Dr. Baeltz Aufnahme. Der Status, den man dort aufnahm, lautet folgendermaßen: Ziemlich gut gebauter Mann, Gesichtsausdruck frei, Hant glatt und elastisch, von normaler Feuchtigkeit und Temperatur. Hände und Füsse sind kalt und cyanostisch. Beim Herabhängen der Füsse tritt die Cyanose an denselben sehr stark hervor. Die Kopfhaare sind dünn; an der Nägeln findet sich keine Abnormität. Das Unterhautfettgewebe ist mässig reduziert, namentlich an den unteren Extremitäten, Adipositas findet sich nirgends.

Die Muskulatur ist im allgemeinen weich, besonders am linken Bein. Knochen und Gelenke normal, doch ist der Bau des Skeletts etwas zart; beim Gehen tritt eine leichte Beckenverschiebung ein und zwar steht die rechte Seite etwas höher und die linke bestrebt sich, sich mit dem linken Femur möglichst in die Vertikale einzastellen, um die Schwerpunktslinie des ganzen Körpers mehr der linken Extremität zu nähern. Sonst keine abnorme Krümmung des Rückens und keine Druckempfindlichkeit und keine Schmerzen an der Wirbelsäule. Die oberflächlichen Nervenstämme sind weder verdickt noch druckempfindlich. Radialarterie nicht sklerosiert. Puls klein, doch gut gespannt. Frequenz 80. Gesicht: Keine Facialislähmung, Augen gut schliessbar, Pupillen normal, Bewegungen der Bulbi sind nach allen Seiten normal. Zunge etwas dünn, weisslich belegt, ohne Zittern, gut beweglich nach allen Seiten hin.

Weicher Gaumen geht beim Phonieren in die Höhe. Der Thorax bewegt sich symmetrisch. Atmung kostoabdominal. Spitzenstoss im sechsten Interkostalraum innerhalb der Mammillarlinie, Herztöne etwas leise. Lungen frei, Leber fühlbar. Triceps- und Handreflex etwas gesteigert. Patellarreflexe beiderseits etwas schwach; Achillessehnenreflexe normal, kein Bauch- und Fusssohlenreflex, mechanische Muskelerregbarkeit überall gesteigert. Sensibilität überall normal; ganz leichte Hypalgesie an der Aussenseite des linken Unterschenkels. Sämtliche Muskeln des Rückens, der Brust und Extremitäten sind atrophisch; besonders ausgeprägt ist dies an dem linken Bein und an dem rechten Arm. In Bezug auf die Stärke der Atrophie folgen sich die einzelnen Körperteile in folgender Weise auf einander: linke Unterschenkel- und linke kleine Fussmuskulatur; rechte Unterschenkel- und rechte kleine Fussmuskulatur; linke Oberschenkelmuskulatur (Benger und Strecker besonders), rechte Obersehenkelmuskulatur (Beuger und Strecker besonders); rechte Schultergürtel-, rechte Oberarm-, rechte Vorderarm-, rechte Pektoralmuskulatur; linke Schultergürtel-, linke Oberarm-, linke Vorderarmmuskulatur; Erector trunei. 
In folgender Tabelle ist die Bewegungsfähigkeit der verschiedenen Muskeln dargestellt.

\begin{tabular}{|c|c|c|}
\hline & Links & Rechts \\
\hline $\begin{array}{l}\text { Hebung des ganzen ge- } \\
\text { streckten } \\
\text { Reickenlage }\end{array}$ & unmöglich & $\begin{array}{l}\text { bis zur Höhe von } 3-4 \\
\text { Zoll möglich }\end{array}$ \\
\hline Bewegung des Knies & $\begin{array}{l}\text { bis } \mathbf{z u} \text { einem rechten } \\
\text { Winkel, aber schwach }\end{array}$ & möglich, aber schwach \\
\hline $\begin{array}{l}\text { Streckung d. Kniee (diese } \\
\text { ist kräftiger als die Beu- } \\
\text { gung) }\end{array}$ & möglich, doch schwach & $\begin{array}{l}\text { möglich und besser als } \\
\text { links }\end{array}$ \\
\hline $\begin{array}{l}\text { Beugung und Streckung } \\
\text { des Fusses }\end{array}$ & kaum möglich & schwach, aber möglich. \\
\hline Beugung der Zehen & schwach & schwach \\
\hline Streckung der Zehen & unmöglich & schwach \\
\hline $\begin{array}{l}\text { Kraft des ersten Inter- } \\
\text { osseus }\end{array}$ & sehr gering & gering \\
\hline Emporheben des Arms & möglich & möglich \\
\hline Adduktion des Oberarms & ziemlich kräftig & ziemlich kräftig \\
\hline Abduktion des Oberarms & schwach & sehr schwach \\
\hline $\begin{array}{l}\text { Beugung in Ellenbogen- } \\
\text { gelenk }\end{array}$ & ziemlich kräftig & etwas schwach \\
\hline $\begin{array}{l}\text { Streckung im Ellenbogen- } \\
\text { gelenk }\end{array}$ & schwach & sehr schwach \\
\hline Beugung im Handgelenk & ziemlich schwach & schwächer als links. \\
\hline Spreizen der F'inger & möglich & möglich \\
\hline Greifkraft der Hand & schwach & schwach \\
\hline Gesichtsmuskeln & frei & frei \\
\hline
\end{tabular}

Der Patient kann sich aus der Rückenlage von selbst aufrichten, jedoch nicht zum Stehen, selbst wenn er sich grosse Mühe gibt und dabei Hände und Arme zu Hilfe nimmt. Hat man ihn aber zum Stehen gebracht, so kann er leidlich vorwärtsgehen, wobei ein Schleppen des linken Beines und eine geringe Beckenverschiebung hervortritt. Der Gang ist breitbeinig und paretisch. Nachdem er einige Male im Zimmer auf- und abgegangen 
ist, verspürt er grosse Müdigkeit und ein Spannungsgefühl in den Waden. Keine ataktischen Symptome.

Weiterer Krankheitsverlauf. 6. Nov. Der rechte Arm wird heute nicht höher. gehoben wie vorher.

9. Nov. Patient gibt an, dass die Arme leicht ermüden. Keine Bulbärsymptome.

12. Nov. Seit einigen Tagen leichte Schmerzen an Hand- und Zehengelenken. Wurmabtreibung: dreizehn Anchylostomen und vier Askariden abgetrieben.

18. Nov. Eine hölzerne Hantel (245-265 g), welche der Patient bei gestrecktem linken Arm ergreift, kann-er bis zur Horizontalen erheben. Nachdem er sie sechzehnmal geschwenkt hat, verspürt er ein Schwächegefühl in den Armen, die Hebung der Hantel mit dem rechten gestreckten Arm bis zur Horizontalen ist ihm nur 30-40 Sekunden möglich.

19. Nov. Heate kann der Patient das linke Knie kaum strecken noch beugen. Die Beugung im Fussgelenk fällt sehr schwer. Die Atrophie der beiderseitigen Unterschenkelmuskulatur hat zugenommen.

23. Nov. Patellarreflex rechts wie bisher, links aber entschieden schwächer geworden. Wenn der Kranke einige Male im Zimmer herumgegangen ist, bekommt er spannende Schmerzen in den Streckern des linken Oberschenkels.

27. Nov. Spannungsgefühl an der linken Rückenseite und den Waden beim Gehen.

29. Nov. Patient kann kaum gehen und droht immer umzufallen. Keine Bulbärsymptome. $A b$ und zu in den Extremitäten fibrilläre Zuckungen, die nachts besonders stark waren.

6. Dez. Reflexe der unteren Extremitäten sind bedentend schwächer, die des Triceps jedoch gesteigert.

9. Dez. Leichte Volumzunahme des linken Arms und der Brust durch Fettansatz. Linke Glutäalmuskulatur atrophischer als die rechte.

11. Dez. Patellarreflex beiderseits sehr schwach; ebenso die Achillessehnenreflexe.

4. Jan. 1902. Die Kraft der beiden Hände ist bedeutend abgeschwächt. Gang sehr erschwert, fast unmöglich.

14. Jan. Gang sehr erschwert, fast unmöglich. Heben des rechten Arms sehr schwierig; Greifkraft stark herabgesetzt.

16. Jan. Tricepsreflex der beiden oberen Extremitäten enorm gesteigert; fibrilläre Zuckungen an beiden Vorderarmen sichtbar.

18. Jan. Triceps brachii und Streckmuskeln des rechten Vorderarms stärker atrophisch als die Beuger. Beide Vorderarme sowie Hände kalt und cyanostisch.

20. Jan. Patient klagt in der letzten Zeit über Müdigkeit in der beiderseitigen Schultergegend.

25. Jan. Triceps und Handreflex auf der linken Seite weit mehr gesteigert als rechts.

3. Febr. Patellar- und Achillessehnenreflex beiderseits sehr schwach. Sehnenreflex an den oberen Extremitäten noch ebenso stark wie früher. Patient fühlt an beiden Beinen fibrilläre Zuckungen.

5. Febr. Die Schwäche der Beine hat sehr bedeutend zugenommen. 
Patient kann ohne Hilfe weder gehen, noch selbst einen Lagewechsel vornehmen.

10. Febr. Leichte Hypästhesie am Rücken, an der Aussen- und Vorderfläche der beiden Oberextremitäten, die nach unten allmählich abnimmt. Waden und Fussmuskeln druckempfindlich. Puls stark gespannt, Herzaktion gesteigert, Herztöne rein.

5. März. Patellarreflex beinahe aufgehoben. Bewegung des linken Beines fast unmöglich.

Patient wird jetzt auf seinen Wunsch entlassen, jedoch am 30. März desselben Jahres in meiner Klinik wieder aufgenommen.

1. April. Äusserlich gut genährter Mann; die Haut elastisch, feucht, aber lederartig dick; das Unterhautfettgewebe überall sehr reichlich entwickelt, so dass der Kranke im Gesicht ein überaus blühendes und wohlgenährtes Aussehen hat. Statur klein, Gesichtsausdruck etwas starr. Conjunctiva palpebralis etwas anämisch, Pupillen normal weit, gleichgross, reagieren auf Licht und bei der Akkomodation gut. Bewegungen der Bulbi nach allen Richtungen frei. Kein Nystagmus. Augenlider gut und kräftig schliessbar; kein Zittern beim Lidschluss. Lippen von normaler Farbe, nicht atrophisch, fest verschliessbar. Das Pfeifen gelingt ohne Mühe. Zunge schmal, rot, nicht belegt. Keine deutliche Atrophie bemerkbar. Bewegungen der Zunge nach allen Richtungen gut; kein fibrilläres Zittern. Tonsillen normal. Weicher Gaumen beiderseits bei der Phonation gleich hoch gehoben. Hals dick und kurz. Thorax ziemlich gut gebaut; Atmung kostoabdominal, von normaler Frequenz. Puls mässig gut gespannt. Pulsfrequenz normal. Radialarterie nicht atheromatös. Herzdämpfung normal, Töne rein; Lungen frei. Bauch mässig aufgetrieben; Leber und Milz nicht fühlbar. Patellar-, Triceps und Achillessehnenreflex nicht vorhanden. Fusssohlen-, Kremaster- und Bauchreflex kaum nachweisbar. Mechanische Erregbarkeit der Muskeln iuberall gesteigert, am rechten Fussgelenk leichte Kontraktur.

Sensibilität überall normal. Die Muskelatrophie ist am stärksten ausgeprägt an den Unterschenkeln, dann folgt die Oberextremitäten- und schliesslich die Handmuskulatur. Die Atrophie der Schulter-, Brust- und Rückenmuskulatur ist durch das reichlich entwickelte Fettpolster teilweise verdeckt.

Die dickste Stelle beträgt in ihrem Umfang

\begin{tabular}{|c|c|c|}
\hline am & rechten Oberarm & $21,0 \mathrm{~cm}$ \\
\hline$\eta$ & linken $"$ & 20,0 \\
\hline$\because$ & rechten Vorderarm & 18,8 \\
\hline & linken $\quad$, & 19,0 \\
\hline an & beiden Oberschenkeln & 35,0 \\
\hline & Unterschenkeln & 20,5 \\
\hline
\end{tabular}

Am Fussgelenk ist eine ganz geringe Beugung und Streckung möglich. Mit den Zehen kann nur eine sehr geringe Plantarflexion gemacht werden. Die aktive Beweglichkeit in beiden Kniegelenken ist fast null. Rotation, Abduktion, Adduktion, Beugung und Streckung im Hüftgelenk gar nicht ausführbar. Die Greifkraft der beiden Hände ist sehr herabgesetzt; Opposition des rechten Daumens unmöglich, die des linken erhalten. Beugung im Handgelenk kaum ausführbar. Beugung und Streckung im Ellenbogen- 
gelenk sehr schwach. Die Adduktion der Oberarme geschieht mit ziemlicher Kraft, während die Abduktion fast unmöglich ist. Suppination und Pronation beider Vorderarme sehr schwer. Die beiden Arme können nur mit grosser Mühe bis zu einer Höhe von $45^{\circ}$ gehoben werden.

In der Rückenlage gelingt es dem Patienten nicht, die Brust mit dem Kinn zu berühren, während dies im Sitzen keine Schwierigkeiten macht. Die seitliche Bewegung des Halses ist nach beiden Seiten ungehindert. Eine Atrophie der Halsmuskeln lässt sich wegen reich entwickelten Fettpolsters nicht erkennen. Die Sitzlage ist dem Patienten ohne Unterstützung nicht möglich, da er gleich nach hinten fallen würde. Eine Umdrehung, ja sogar die geringste Bewegung des Rumpfes kann er nicht machen. Bei halb erbobener Rückenlage ist der Bauch weich. Beim Husten sowie bei Defäkation ist die Banchpresse fast null. Die Wirbelsäule ist gerade und nirgends druckempfindlich.

Elektrische Prüfung der Nerven und Muskeln. Am N. ulnaris, radialis und medianus lässt sich eine leichte Herabsetzung der galvanischen und faradischen Erregbarkeit konstatieren. Der N. peroneus ist unerregbar durch den faradischen und galvanischen Strom, wäbrend beim N. tibialis die Erregbarkeit durch diese beiden Stromarten stark herabgesetzt ist. In mehr oder minder hohem Grade ist die Erregbarkeit in allen Oberschenkelmuskeln gegenüber beiden elektrischen Stromesarten herabgesetzt. Träge Zuckung. und Herabsetzung der elektrischen Erregbarkeit des linken M. biceps, der beiderseitigen $\mathrm{Mm}$. fl. carp. radial, M. abd. poll. brev. sin., M. abd. digit. quinti, M. flex. digiti quinti, M. lumbricalis II. Unerregbar gegen beide Stromarten sind beiderseits die M. sartorius, Adduktoren, M. tibialis, ant., M. exts. digit. comm. long. und M. peroneus. Der M. gastrocnemius zeigt starke Herabsetzung der elektrischen Erregbarkeit mit Umkehr der Zuckungsformel. Ebenso verhalten sich die Musculi interossei.

Weiterer Krankheitsbericht: 2. Nov. Seit einiger Zeit kann Patient die Zeitung im Liegen nicht mehr lange halten.

14. Nov. Die Streckung der Hände macht grössere Mühe als die Beugang. Die Spreizung der Finger ist möglich, geschieht jedoch ungleichmässig und langsam; man beobachtet dabei an den Fingern kleinwelliges Zittern. Die Opposition der Daumen, die gegen die kleinen Finger unmöglich ist, gelingt im übrigen mit grosser Mühe.

Die Supination der Hände ist total unmöglich and die Pronation erfolgt nur in sehr beschränktem Maße. Nach hinten kann Patient den Kopf nur wenig bewegen, nach vorne überhaupt nicht. Die Arme vermag er fast gar nicht mehr zu erheben. Abgesehen von einer ganz geringfügigen Bewegungsfähigkeit in den Fussgelenken sind die Unterextremitäten völlig gelähmt. Das rechte Bein zeigt bei passiven Bewegungen eine leichte Kontraktur in allen Gelenken. Diese fehlt bei den Oberextremitäten. Die Reflexe sind sämtlich aufgehoben. Die Bauchpresse kommt so gut wie nicht mehr zustande.

12. Dez. Seit gestern stellte sich kraftloses Hüsteln ein, durch welches spärliche Sputa nur mit grosser Mühe herausbefördert wurden. Ferner trat ein allgemeines Unbehagen und Schmerzhaftigkeit der ganzen Muskulatur auf. Ausserdem klagt Patient über Frostgefühl und Kopfschmerzen. Abends 6 Uhr stellt sich starke Dyspnose, Cyanose und Erstickungsgefühl ein, da die Sputa aus der Trachea und dem Larynx nicht mehr heraus- 
befördert werden können. Der ganze Körper bedeckt sich mit kaltem Schweiss. Patient wird fast bewusstlos und entleert unwillkürlich Urin und Kot. Auskultatorisch bemerkt man am ganzen Thorax trockenes Rasseln: Atemgeräusch rechts etwas schwächer als links. Keine Dämpfung. Stimmfremitus überall vorhanden. Die Temperatur stieg nachmittags plötzlich auf $38,7^{\circ}$; Puls 115 . Unterleib aufgetrieben. Man leitet die künstliche Atmung ein, die die ganze Nacht fortgesetzt wird.

13. Dez. Vormittags ist die Dyspnoe etwas geringer, jedoch immer noch stark ausgeprägt, so dass man die künstliche Atmung weiter fortsetzt. Der Husten ist sehr kraftlos, die Stimme seit gestern aphonisch. Der Schluckakt ist zwar ungestört, doch verweigert der Patient die Nahrungsaufnahme wegen der hochgradigen Atemnot. Puls 150, relativ gut gespannt. Temperatur $37,2^{\circ}$.

Um 10 Uhr vormittags wird die Dyspnoe sehr hochgradig und Gesicht sowie Extremitäten zeigen starke Cyanose. Der Thorax, der sich bei der Atmung gar nicht erweitert, wird nur gehoben und gesenkt. Puls klein und schwach. Pupillen sind eng und reagieren fast nicht mehr auf Lichtreiz. Die Cyanose, die nur durch forcierte künstliche Atmung zum Verschwinden gebracht wird, tritt beim Unterlassen derselben sofort wieder hervor. Bei jeder Inspiration wird der Mund weit geöffnet und der Kopf nach hinten geworfen. Die Nasenflügel beteiligen sich an der Atmung.

14. Dez. Das Leben wird nur vermittelst künstlicher Atmung erhaIten, welche die ganze Nacht hindurch fortgesetzt wurde. Sprache ist wegen der hochgradigen Dyspnoe kraftlos, unterbrochen und fast unverständlich. Pupillen grösser als gestern, reagieren nicht auf Lichteinfall. Temperatur $36,0^{\circ}$, Puls kaum fühllbar. Bauch stark aufgetrieben. Sensorium klar.

Um 1 Uhr nachmittags wird der Zustand sehr schlecht. Weder künstliche Atmung noch Kampherinjektionen vermögen zu helfen. Puls kaum fühlbar, Herztöne sehr leise, Sensorium klar, Sprechen unmöglich. Über beiden Lungen überall trockenes Rasseln, sehr starke Cyanose.

11/2 Uhr nachmittags Puls nicht mehr fühlbar, Herztöne leise, doch regelmässig, Pupillen mittelweit.

Um $3^{3 / 4}$ Uhr nachmittags erfolgt der Tod.

Nach 18 Stunden wurde die Sektion gemacht.

Sektionsbefund. Männliche Leiche, mässig ausgeprägte Totenstarre. Am Rücken diffuse Leichenflecke. Fettpolster an Brust und Bauch, sowie der hinteren Halsgegend kolossal entwickelt. An letzterer Stelle beträgt die Dicke des Fettpolsters etwa $2 \mathrm{~cm}$, am Bauch 2,5 cm. Die Rückenmuskeln sehen etwas blass aus, zeigen aber keine interstitielle Fettwucherung.

Rückenmark: Dura mater spinalis normal, Pia mater an der Dorsalseite überall stark injiziert, besonders ist dies am Lendenteil ausgeprägt. Konsistenz normal. Die Schnittfläche feucht und glänzend. Konfiguration der grauen Substanz etwas unregelmässig, Vorderhörner stark eingezogen und etwas rötlich verfärbt. Weisse Substanz quillt aus der Schnittfiäche hervor.

Pektoralmuskeln atrophisch, blassrötlich-gelb. Baucheingeweide normal gelagert, Fettgewebe im Bauchraum sehr reichlich entwickelt. Magen und Darm mässig aufgebläht. Blase zusammengezogen. Zwerchfellstand: 
rechts 3., links 5. Rippe. Vorderes Mediastinalfettgewebe sehr reichlich entwickelt. Beide Plenrahöhlen normal. Innenfläche des Perikards glatt und hyperämisch. Herz etwas gross. Subperikardiales Fettgewebe reichlich entwickelt; rechter Vorhof gefüllt mit dunkelrötlichem geronnenen Blut. Rechter Ventrikel enthält mässige Mengen Speckgerinnsel, Muskulatur blass, diffus leicht getrübt, Klappenapparate intakt und zart. Im linken Ventrikel spärliches Blutgerinnsel. Muskulatur blass, leicht getrübt. Aortenlklappen und Aorta zart.

Linke Lunge: Viele Ekchymosen an der Pleura des Unterlappens. Unterlappen atelektatisch. Oberlappen lufthaltig.

Rechte Lunge: Pleura pulmonalis mit zahlreichen Ekchymosen. Oberlappen lufthaltig, Mittellappen atelektatisch. Unterlappen zeigt zahlreiche katarrhalisch-pneumonische Herde. Bronchialschleimhaut hyperämisch und mit dickem Schleim bedeckt. Milz normal. Schädelknochen normal. Aussenfläche der Dura des Gehirns stark injiziert, besonders in den hinteren Partien. Pia am Scheitelteil leicht fibrös, Gefässe derselben stark gefüllt.

Gehirn: Konsistenz normal, Schnittfläche feucht und glänzend, mit zahlreichen Blutpunkten. Im übrigen ohne sichtbare Veränderungen.

Nieren normal, Leber blutreich, Magen katarrhalisch.

Jejunum leicht schiefrig verfärbt. Dasselbe findet sich an den Follikeln des Kolon.

Wadenmuskulatur, Triceps, Biceps und Vorderarmmuskeln sulzig, gelbrötlich und sehr atrophisch. Kleine Handmuskeln blassrötlich und ziemlich atrophisch.

Die Nn. radialis, medianus, peronens und phrenicus weisslich und, wie es scheint, etwas dünner als normal. N. vagus von normalem Aussehen.

Mikroskopischer Befund des Nervensystems und der Muskulatur: Medulla oblongata und Rückenmark: Zur Färbung der Schnitte wurden verwendet die Methoden von van Gieson, Weigert, Marchi und die Karminfärbung.

Medulla oblongata in der Höhe der Schleifenkreuzung: In der Bahn der Schleifen- und Pyramidenkreuzung sowie der Kleinhirnseitenstrangbahn sieht man durch Osmium geschwärzte Massen zerstreut. Die Ganglienzellen am ventralen Teile des Zentralkanals scheinen normal zu sein. Die Gefässe in der granen Substanz sind gefulltt.

Die im oberen Teile der Medulla oblongata liegenden Hypoglossusund Vaguskerne sind normal, ebenso ihre intra- und extramedullären Wurzeln. Die Gefässe der Rautengrube sind gefüllt.

Höhe der Pyramidenkreuzung: In der Kleinhirnseitenstrangbahn und der Pyramidenkreuzung sieht man zerstreute Massen, die durch die Marchische Methode geschwärzt sind. Dabei sind die in der Pyramidenkreuzung liegenden Massen den Nervenfasern parallel angeordnet. Im Vorderhorn sind die Ganglienzellen spärlich, doch sieht man einige wohlausgebildete. Die Gefässe sind gefüllt.

Höhe des mittleren Cervikalmarkes: Überall in der weissen Substanz, einschliesslich der vorderen Kommissur, findet man durch Osmium schwarzgefärbte zerstreute Massen. Spärlicher sind diese im Hinterstrang und der grauen Substanz. Die vorderen Wurzeln enthalten noch viele normale Fasern, doch lässt sich ein Ausfall einer ganzen Reihe von Fasern 
nachweisen. Die markhaltigen Fasern im Vorderhorn sind zahlreich, jedoch fast alle kuiz und abgebrochen. Im Vorderstranggrundbündel sowie wenigstens etwas in der ganzen Peripherie des Seitenstranges ist geringer Faserausfall und dementsprechende Neurogliawucherung zu beobachten. Die Vorderhornganglienzellen sind meistens verschwunden, doch finden sich daneben mehrere atrophische und wenig normale Zellen. Die Spinnenzellen sind zahlreich. Die Gefässe sind verdickt und zellig infiltriert. Viele neugebildete Gefässe sind sichtbar.

In der Höhe des unteren Cervikalmarkes ergiebt sich derselbe Befund wie oben.

Im oberen Brustmark Vorderhornganglienzellen fast verschwunden, nur einige normale Ganglienzellen noch sichtbar. Im Vorderstranggrundbündel diffuse bis an die Peripherie reichende, ganz mässige Neurogliawucherung mit entsprechendem Faserausfall. Die durch Osmium geschwärzten Massen sind zerstreut sichtbar. In der Kommissur stellen sie längliche, rosenkranzförmige Ketten dar. In der grauen Substanz und dem Hinterstrang sind sie in geringer Anzahl vorhanden. Die Markfasern sind in der grauen Substanz kurz und abgebrochen; Spinnenzellen spärlich, Neurogliakerne zahlreich. Gefässe nicht verdickt. Die Clarkeschen Säulen sind normal und darin weder Zellen noch Fasern verändert.

Mittleres Dorsalmark: Ganglienzellen fast verschwunden, doch sind einige normale noch sichtbar. Die durch Osmium geschwärzten Massen findet man zerstreut, sie sind in der grauen Substanz und im Hinterstrang spärlich; in der vorderen Kommissur findet man sie länglich und längs den Nervenfasern angeordnet. Die vorderen Wurzeln enthalten zahlreiche normale Fasern, doch sind viele Faseransfälle zu konstatieren. Die Markfasern des Vorderhorns sind kurz und abgebrochen, Gefässe nicht verdickt. $\mathrm{Cl}$ arkesche Säule normal. Im Vorderstranggrundbündel um das Vorderhorn herum leichter Faserausfall mit dementsprechender Neurogliawucherung.

Unteres Dorsalmark: Die durch Osmium geschwärzten Massen liegen zerstreut in der ganzen Schnittfläche, aber im Hinterstrang und der grauen Substanz sind sie spärlich. In der vorderen Kommissur stellen sie sich als längliche Massen dar. Die Vorderhornganglienzellen sind fast verschwunden und man sieht nur noch einige normale. Die Gefässe sind gefụllt und nicht verdickt. Markfasern im Vorderhorn kurz und abgebrochen. Die vorderen Wurzelfasern sind mässig ausgelichtet. Der Faserausfall im Vorderstranggrundbündel ist nur mässig. Clarkesche Säulen normal.

Lendenmark: Die Ganglienzellen sind fast verschwunden, nur einige sehr atrophische, doch fast gar keine normalen mehr sichtbar. Die Spinnenzellen sind kolossal vermehrt, die Gefässe verdickt, kleinzellig infiltriert und zahIreich neugebildet. Sämtliche Gefässe zeigen Injektion; Vorderwurzeln atrophisch; sehr viele Fasern ausgefallen. Bei der Marchischen Färbung sieht man auf der ganzen Schnittfläche schwärzliche Massen, die nur in der grauen Substanz and der vorderen Kommissur spärlich sind. Im Vorderstranggrundbündel and dem Seitenstrang sind die Nervenfasern ziemlich reichlich ausgefallen, dafür ist Neurogliawucherung eingetreten. Das Gebiet der gekreuzten Pyramidenbahn ist auch sklerosiert. Die Markfasern im Vorderhorn sind kurz abgebrochen, doch findet man schöne lange Nervenfaserbündel. 
Sakralmark: Die Ganglienzellen der Vorderhörner sind grösstenteils geschwunden, nur die mediale Zellgruppe ist beiderseits relativ gut erhalten und nur einige davon atrophisch. Die Gefässe sind zahlreich und stark gefüllt, ihre Wände verdickt und zellig infiltriert. Die Spinnenzellen in den Vorderhörnern sehr vermehrt. Die markhaltigen Fasern der Vorderhörner sind kurz und abgebrochen, sowie spärlicher als normal, doch erkennt man eine ziemlich grosse Menge von gut ausgebildeten Nervenfaserbündeln. In der weissen Substanz, mit Ausnahme des Hinterstranges, tritt ein ziemlich beträchtlicher diffuser Faserausfall mit entsprechender Neurogliawucherung hervor. Vorderwurzeln atrophisch, mit spärlichen normalen Nervenfasern. Neuroglia vermehrt.

Periphere Nerven: N. vagus und recurrens normal.

Beim N. radialis und medianus ist der Faserausfall gering; die Gefässe sind gefüllt. N. phrenicus zeigt ziemlich bemerkbaren Faserausfall, was beim $\mathrm{N}$. peroneus weniger hervortritt, ziemlich stark aber wieder an den intramuskulären Nervenfasern (Wadenmuskeln).

Die Rücken-, Waden- und Oberarmmuskeln weisen zahlreiche atrophische Fasern auf, deren Querstreifung überall hervortritt. Die Sarkolemmkerne sind stark vermehrt und einzelne atrophische Muskelfasern zeigen dichotomische Teilungen. Das interstitielle Fett- und Bindegewebe ist wenig vermehrt.

Zwerchfellmuskulatur: Neben einer mässigen Zahl atrophischer Muskelfasern findet man lauter homogen degenerierte. Die Querstreifung fehlt ibberall, Sarkolemmkernwucherung besteht nicht.

Die Daumenballenmuskulatur ist weniger verändert. Die atrophischen Muskelfasern sind viel spärlicher als zum Beispiel an den Wadenmuskeln. Man findet eine mässige Vermehrung der Sarkolemmkerne.

Die Zungenmuskulatur ist normal.

Wir wenden uns jetzt zur Besprechung des eben mitgeteilten Falles. Wie man ohne weiteres sieht, handelt es sich hierbei um eine reine Vorderhornerkrankung. Kliniseh besonders bemerkenswert ist dabei die anfängliche Lebhaftigkeit des Tricepsreflexes, weswegen die Diagnose anfangs erschwert wurde.

In einer grossen Reihe der bis jetzf beobachteten Fälle von Poliomyelitis anterior chronica findet man die Lebhaftigkeit der Sehnenreflexe und man hat sie auf verschiedene Weise zu deuten versucht. Redlich berichtet von einem Fall mit beiderseitiger Steigerung der Patellarreflexe. Dasselbe gilt ron einem Falle Erbs. Ewald fand Steigerung des Patellar- und Achillessehnenreflexes; der Fussklonus war nur angedeutet. Auch Schuster spricht von einer bedeutenden Steigerung des Patellarreflexes; endlich hat Senator einen Fall beschrieben, in dem sich das klinische Bild der amyotrophischen Lateralsklerose fand, ohne dass man später bei der Autopsie eine Degeneration der Pyramidenbahn hätte nachweisen können, und gelegentlich der Demonstration dieses interessanten Falles machte Leyden die geistreiche Bemerkung, dass zum Zustandekommen der spezifischen 
Erscheinungen nicht die Degeneration bestimmter Faserzüge nötig sei, sondern dass diese Symptome auch deshalb auftreten können, weil der Willensimpuls vom Gehirn nicht bis zu den Muskeln hingelangt. In den Fällen, wo das letztere zutreffe, überwiege dann die Innervation durch die Reflexleitung, und wenn eben die Muskeln nicht vollständig atrophiseh seien, gebe sich eine Steigerung der Reflexe in einer Steigerung des Tonus der Muskeln, also in spastischen Erscheinungen zu erkennen.

Nach Erb erklärt sich die Lebhaftigkeit der Reflexe unserer Krankheit durch das Weiterfortschreiten der Degenerationsprozesse in in den Vorderhörnern, das mit leichten Irritationserscheinungen einhergehe.

Schuster zieht zur Erklärung der gesteigerten Patellarreflexe die in seinem Falle gleichzeitig beobachtete Seitenstrangerkrankung heran. In meinem Falle lag eine solche nicht vor, so dass mir die Erbsche Erklärung die zutreffende zu sein scheint.

Bei allen bis jetzt beobachteten Poliomyelitisfällen, die mit lebhafter Reflexsteigerung einhergingen, hat man, abgesehen von dem Senatorschen Falle, niemals spastische Erscheinungen oder echten Fussklonus beobachtet, so dass die Differentialdiagnose gegenüber amyotropher Lateralsklerose meist leicht zu stellen war. Dieselbe ist natürlich sehr schwierig oder unmöglich bei Fällen von amyotrophischer Lateralsklerose, in denen die Sehnenreflexe abgeschwächt sind oder gänzlich fehlen.

Kahler erklärt das Phänomen der spastischen Symptome, indem er annimmt, dass die Degeneration sich im Beginn der Erkrankung vorwiegend auf die motorischen Bahnen der weissen Substanz beschränke und später erst auf den motorischen Teil der grauen übergehe. Bei geringer Entwicklung oder Fehlen der Spasmen seien beide Gebiete gleichzeitig oder in der eben angegebenen umgekehrten Reihenfolge von der Degeneration ergriffen.

In neuerer Zeit haben hervorragende Forscher die Ansicht geäussert, dass man die progressive spinale Muskelatrophie der amyotrophischen Lateralsklerose zurechnen müsse; doch hiergegen wendet sich J. B. Charcot in seiner ausführlichen Monographie, in der er überzeugend darlegt, dass beide ein für sich wohlcharakterisiertes Krankheitsbild bieten und durchaus zu trennen sind. Andererseits ist wieder von kompetenter Seite daran gezweifelt worden, dass die Poliomyelitis anterior chronica mit der progressiven spinalen Muskelatrophie vom Typus Aran-Duchenne zu identifizieren sei. J. B. Chareot neigt sich dieser Ansicht zu, indem er meint, dass beide Krankheiten etwa in dem Verhältnis zu einander stünden wie die galoppierende 
Phthise zur chronischen Lungentuberkulose. Es möge in dieser Beziehung noch das Folgende hervorgehoben werden: Einige Forscher, z. B. R. Ewald und Bielschowsky meinen, dass das Vorhandensein verdickter Gefässe und Blutungen bei der Erkrankung der Vorderhornzellen auf einen entzündlichen Vorgang schliessen lasse und für die Poliomyelitis anterior chronica charakteristisch sei, dass es sich aber beim Fehlen solcher Gefässveränderungen um eine progressive spinale Muskelatrophie handle; doch ist durch Schmaus bereits nachgewiesen worden, dass die Gefässverdickungen zu inkonstant sejen, um sie differentialdiagnostisch zu verwerten. In dem Senatorschen Falle fanden sich trotz der Integrität des Gefässapparates Blutaustritte in der grauen Substanz, die zum Teil älteren, zum Teil jüngeren Datums waren. Senator führt ihr Zustandekommen auf terminale kachektische Erscheinungen zurück. Die Gefässveränderungen in unserem Falle fanden sich nur am Hals-, Lenden- und Sakralmark, dort, wo die Destruktion der grauen Substanz am meisten vorgeschritten war. Im ganzen Dorsal- und oberen Halsmark waren die Gefässe zwar stark gefüllt, doch fehlten jegliche anatomischen Veränderungen. Ich bin der Meinung, dass hier die Gefässveränderungen nur als sekundär aufzufassen sind. Nach der Ansicht von Schmaus ist ja der Gefässerkrankung bei der progressiven spinalen Muskelatrophie weiter keine Bedeutung beizumessen.

Was nun die klinischen Unterschiede des weiteren angeht, so ist nach Strümpell folgendes zu sagen:

Bei der progressiven spinalen Muskelatrophie geht langsam Faser nach Faser zugrunde und der Atrophie geht die Muskelschwäche vollkommen parallel, während bei der chronischen Poliomyelitis die Lähmung der Atrophie vorausgeht und von vornherein ganze Muskeln, resp. Muskelgebiete befällt. Hierzu sagt Göbel folgendes: „Differentialdiagnostisch wird bei der progressiven spinalen Muskelatrophie gegenüber der Poliomyelitis anterior chronica stets hervorgehoben, dass der Beginn der Atrophie in unmerklicher Weise der subjektiven und objektiven Schwäche vorangebe. Man hat dies jedoch wohl cum grano salis zu verstehen, denn es handelt sich bei Beurteilung dieser Frage doch sowohl um eine feine Beobachtung seitens des Patienten, als besonders um ein frïhzeitiges Erkennen durch den Arzt."

Das Auftreten der progressiven spinalen Muskelatrophie ist mit wenigen Ausnahmen auf das vorgerücktere Alter beschränkt (nach Strümpell im 40.-50. Lebensjahre); etwas ähnliches gilt von unserer Krankheit, die man von dem 36.-53. Lebensjahre beobachtet hat. Allerdings ist ein Fall von Bielschowsky bekannt, in dem die Erkrankung schon im 8. Lebensjahre ihren Anfang nahm; auch andere 
derartige Fälle sind beschrieben. Der erste, der das Auftreten von Poliomyelitis anterior chronica im Kindesalter beobachtete, war Erb. Die von Werdnig und Hoffmann gefundene hereditäre Form rechne ich natürlich nicht hierher.

Es sei jetzt noch einiges über die Dauer des Leidens gesagt; dass unsere Krankheit rascher als die progressive spinale Muskelatrophie verläuft, ist ja allgemein bekannt.

Die Dauer des Krankheitsverlaufes schwankt im allgemeinen zwischen 10 Monaten (Grunow) und 3 Jahren (Ewald). Rechnen wir dann noch den Fall von Bielschowsky, bei dem ja auch die Lähmung der Atrophie vorherging, hinzu, so ergibt sich als Maximum für die Dauer unserer Krankeit 9 Jahre.

Ich möchte noch hinzufügen, dass mein Patient eine Zeit lang das Bild des sich Aufrichtens an den eigenen Beinen dargeboten hat, wie es für die myopathische Form der Muskelatrophie typisch ist, und es ist interessant, dass Charcot in seinem Falle dasselbe beobachtet hat; wir finden hierüber bei ihm die Angabe: „Pour se relever de la position accroupie, il est obligé d'appuyer ses mains sur ses cuisses."

Was nun den pathologisch-anatomischen Befund angeht, so handelt es sich in unserem Falle hauptsächlich um einen Schwund der Ganglienzellen der Vorderhörner vom untersten Sakralmark bis zum obersten Halsmark.

Ein gruppenweises Befallenwerden der Ganglienzellen von der Degeneration liess sich nicht konstatieren. Die Zelldegeneration war überall diffus, nur fiel mir auf, dass im Sakralmark die mediale Zellgruppe fast vollkommen erhalten war, während die anderen Zellgruppen sich absolut nicht mehr wahrnehmen liessen. Wie bei fast allen bis jetzt veröffentlichten Fällen, so fand sich auch in dem meinigen ein mehr oder minder mässiger Faserausfall im Vorderstranggrundbündel, resp. stellenweise im Seiten- und Türkschen Strang. In mehreren Fällen (Oppenheim, Nonne, Ewald etc.) liess sich sogar eine Faserdegeneration im Hinterstrang nachweisen, was die betreffenden Autoren aber doch nicht hinderte, an der anatomischen Diagnose Poliomyelitis festzuhalten.

Ich habe mich bei der Untersuchung des Rückenmarks zur Färbung der Schnitte teilweise der Marchischen Methode bedient, da nach den Darlegungen von Hoche sich hiermit gewisse Fasernveränderungen auch bei alten Rückenmarksaffektionen nachweisen lassen, und auf diese Weise eigenartige Bilder bekommen. Man sah nämlich auf Schnitten, die verschiedenen Rückenmarkshöhen angehörten, diffus zerstreute, schwarze, spitzige, unregelmässig gestaltete Massen, die meiner Meinung nach nicht als ein zufälliger Befund aufgefasst wer- 
den dürfen. Dafür sprechen nämlich die folgenden Tatsachen: Erstens einmal finden sich diese schwarzen Massen nur in geringer Menge in der grauen Substanz und im Hinterstrang, während man sie im Vorder- und Seitenstrang stets in beträchtlicher Menge antrifft. Ferner aber haben sie in der vorderen Kommissur der horizontalen Richtung der dort verlaufenden Nervenfasern entsprechend, diesen parallel gestellt, eine unregelmässig rosenkranzförmige Gestalt angenommen. In der Medulla oblongata sind sie ausschliesslich auf die Pyramidenkreuzung erster und zweiter Ordnung und die Kleinhirnseitenstrangbahn lokalisiert.

Eine Verdickung der Pia mater, welche Oppenheimer und Ewald in ihren Fällen sehr stark ausgesprochen fanden, habe ich in meinem Falle nicht nachweisen können.

Ich füge noch hinzu, dass Verdickung und Zellinfiltration der Gefässwände von mir nur im Hals-; Lenden- und Sakralteil gefunden wurden. In allen anderen Rückenmarksteilen waren die Gefässe zwar wie überall gefüllt, aber frei von jeglichen Veränderungen.

Zum Schluss ist es mir eine angenehme Pflicht, Herrn Professor Dr. Hoffmann in Heidelberg meinen aufrichtigen Dank auszusprechen für die Liebenswürdigkeit, mit der er mich bei der Durchmusterung der Präparate unterstützte, und für manchen wichtigen Wink, den er mir erteilte.

\section{Literatur.}

1) Oppenheim, Über die alkute spinale Erkrankung bei Erwachsenen und über verwandte spinale Erkrankungen. Westphahls Arch. Bd. 5.

2) Derselbe, Pathologie der chronischen atrophischen Spinallähmung. Westphals Arch. Bd. 24.

3) Nonne, Klin. u. anatom. Untersuchung eines Falles von Poliomyelitis ant. chron: Deutsche Zeitschr. f. Nervenheilkde. Bd. I.

4) Darkschewitsch, Ein Fall von chronischer Poliomyelitis. Neurolog. Zentralbl. Bd. XI.

5) Erb, Ūber Modifikation der partiellen Entartungsreaktion und das Vorkommen der chronischen atrophischen Spinallähmung beim Kinde. Neurolog. Zentralbl. 1883.

6) Derselbe, Über Poliomyelitis anterior chronica nach Trauma. Deut. Zeitschr. f. Nervenheilkde. Bd. XI.

7) Lokwood, Report of a peculier case of chron. ascending poliomyelitis ant. New-York. med. Journ. 1892.

8) J. B. Charcot et Dutiel, Note sur une cas de poliomyélite antérieuse chr. suivie d'autopsie. Progrès médical 1894. 
9) J. B. Charcot, Contribution a l'étude de l'atrophie musculaire progressive type Aran-Duchenne.

10) Paul Schuster, Poliomyelitis ant. chron. mit Beteiligung der hinteren Wurzeln und der Burdachschen Stränge. Neurol. Zentralbl. 1897.

11) Redlich, Wiener med. Presse. 1899.

12) Bielschowsky, Zur Histologie de̊r Poliomyelitis ant. chron. Zeitschr. f. klin. Medizin. Bd. 37 .

13) R. Ewald, Ein Fall von Poliomyelitis ant. chron. Inaug.-Diss. 1899.

14) Grunow, Poliomyelitis ant. chron. und acuta der Erwachsenen. Deut. Zeitschr. f. Nervenheilkde. Bd. 20.

15) Oppenheimer, Ein Beitrag zur Pathologie der progr. Muskelatrophie. Inaug.-Diss. 1900.

16) Senator, Ein Fall von sog. amyotrophischer Lateralsklerose. Deutsche med. Wochenschr. 1894.

17) Kahler, Deutsche Zeitschr. f. Heilkde. Bd. 5.

18) Etienne, Nouv. Journ. de la Salpêtrière 1899. Neur. Zentralbl. 1900.

19) Eoche, Znr Pathologie der bulbärspast., spastisch atrophischen Lähmungen. Neurol. Zentralbl. 1897.

20) Placzek, Virchows Arch. Bd. 158.

21) Schmaus, Lehrb. der patholog. Anatomie des Zentralnervensystems.

22) Derselbe, Lubasch-Ostertags Ergebnisse. 1. und 5. Jahrgang.

23) Göbel, Monatsschrift für Psychiatrie und Neurologie. Bd. 3 und 4. Zuammenfassendes Referat über die progr. spinale Muskelatrophie.

24) Villers, Un cas d'atrophie musculaire progr. du type Aran-Duchenne. Journal medic. de Bruxelles. 